\title{
Nomogram for determining the diameter of drainage tiles
}

\author{
A. D. KUSSE
}

Research Dept. of the Koninklijke Nederlandsche Heidemaatschappij, Arnhem, Netherlands

\section{Introduction}

The diameter of a drain is determined by the amount of water to be discharged and the permissible slope, added to which there may be certain requirements with regard to maximum and minimum flow velocities. The discharge per metre of tile is determined by the drainage per unit of area multiplied by the drain spacing.

A number of formulae are known for the flow of water through drain tiles, the effect of the roughness of the walls usually being determined by experiment. In this article we shall not discuss the usefulness of any of these formulae but will construct a nomogram enabling the effect on diameter of such various factors as distance, length, discharge intensity, approximate velocities and slopes and their mutual influence to be read off immediately.

Our starting point is the frequently used formula found empirically by VISSER (1954):

$$
\frac{\mathrm{dh}}{\mathrm{dl}}=-6,8 \times 10^{-4} \frac{\mathrm{v}^{1,818}}{\mathrm{v}^{1,221}}
$$

In the nomograms hitherto used for determining tile diameter (VISSER, 1954) it was possible to define the diameter for a given slope independently of the size of the area to be drained and the discharge intensity.

These nomograms give no information on flow velocity; moreover a separate nomogram is required for each gradient and the length of a line has to be calculated separately. In 1954, VISSER proposed the use of a working chart from which one could read off the relationship between piezometric head and flow velocity. But this working chart can only be used for a particular case. The working chart for each composite drainage system has to be drawn up with the aid of three nomograms. The present article will indicate how all these variables can be shown in a single nomogram, enabling the relationship between drainage length, tile diameter, flow velocity, piezometric slope and discharge intensity to be read off in a simple manner. Other formulae than VISSER's can be used for composing such a nomogram.

\section{The composition of the nomogram}

VISSER gives the following equation for the flow of water through clay tiles:

$$
i_{p}=6,8 \times 10^{-4} \frac{v^{1,818}}{v^{1,221}}
$$

Received for publication 21st February, 1963.

Neth. J. agric. Sci., Vol. 12 (1964) No. 2 (May) 
in which $i_{p}=-\frac{d h}{d l}=$ piezometric slope;

$\mathrm{v}=$ flow velocity in $\mathrm{m} \cdot \mathrm{sec}^{-1}$;

$\mathrm{d}=$ tile diameter in $\mathrm{m}$.

This formula is for water transport. In the case of laterals and collectors the amount of water to be drained increases in the direction of flow. The calculation of the required total piezometric head of a drain, in which water is taken up over the entire length of the line, is obtained by integrating equation (1) with respect to the length, $\mathrm{v}$ being regarded as a function of 1 .

The following equation is valid for every cross-section of a drain :

$$
\mathbf{v}=\frac{\mathrm{q}}{1 / 4 \pi \mathrm{d}^{2}}=\frac{\mathrm{mbl}}{1 / 4 \pi \mathrm{d}^{2}}
$$

in which $\mathrm{d}=$ tile diameter in $\mathrm{m}$;

$\mathrm{b}=$ width of the (parcel) or drain spacing in $\mathrm{m}$;

$1=$ length of the line in $\mathrm{m}$;

$v=$ flow velocity in $\mathrm{m} \cdot \mathrm{sec}^{-1}$;

$\mathrm{m}=$ discharge intensity in $\mathrm{m} \cdot \mathrm{sec}^{-1}$.

$\mathrm{q}$ denotes the discharge and is equal to the discharge intensity multiplied by the area to be drained belonging to a length of the drain 1 . The flow velocity $v$ is the average velocity over a cross-section of a drain at a distance 1 with regard to the beginning of the drain.

This flow velocity substituted in (1) gives :

$$
\frac{\mathrm{dh}}{\mathrm{dl}}=-6,8 \times 10^{-4}\left(\frac{4}{\pi} \mathrm{mbl}\right)^{1,818} \cdot \mathrm{d}^{-4,857}
$$

After integrating the equation and making use of the geometric slope $i_{g}=-\frac{h}{1}$ in which ( $-\mathrm{h}$ ) represents the decrease in height of the drainage tile in the direction of flow over a total length 1 , we get: $\mathrm{mbl}=76,6 \mathrm{~d}^{2,672}, \mathrm{i}_{\mathrm{g}}{ }^{0,55}$.

If $i_{g}$ is expressed in $\%, \mathrm{~d}$ in $\mathrm{cm}, \mathrm{b}$ and $\mathrm{l}$ in metres and $\mathrm{m}$ in $\mathrm{mm}(24 \mathrm{hrs})^{-1}$, the equation can be written as follows:

$$
\mathrm{mbl}=2408 \mathrm{~d}^{2,672} \cdot \mathrm{i}_{\mathrm{g}}{ }^{0,55}
$$

or: $\log \mathrm{mb}=\log 2408+2,672 \log \mathrm{d}+0,55 \log \mathrm{i}_{\mathrm{g}}-\log 1$.

If $i_{g}$ is assumed to be constant and $m b$ is plotted on logarithmical paper against 1 , a straight line can be drawn for each value of $d$, the tangent of the angle of slope of which is -1 (see FIG. 1). Straight lines in the tangent -1 can also be drawn for various piezometric slopes and a constant value of $d$.

If, for example, in a tile diameter the length for a certain mb-value is $l_{1}$ for a slope of $0,10 \%$, the length $l_{2}$ for a slope of $0,20 \%$ with the same tile diameter is then

$$
\frac{0,200,55}{0,10^{0,55}} \times 1_{1}=1,464 l_{1}=l_{2}
$$

This horizontal displacement with respect to the length 1 is now converted to a ver- 


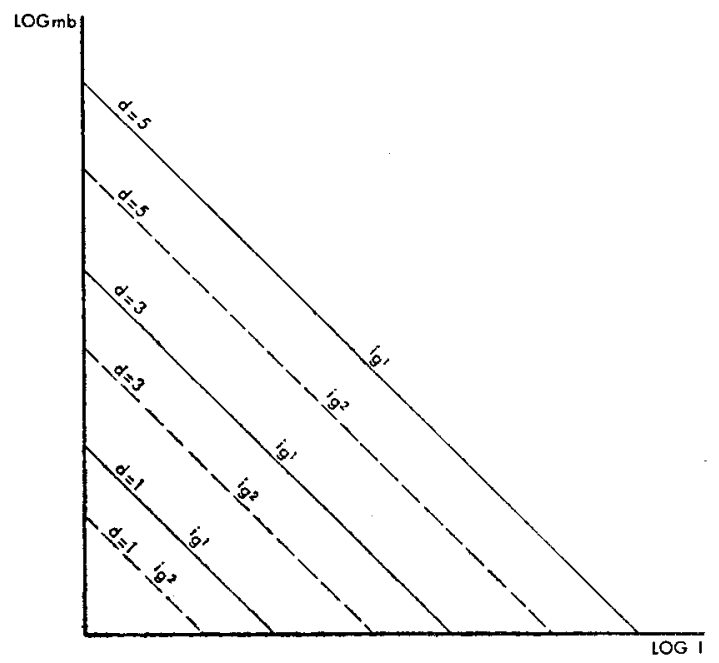

Fig. 1

The relationship between $\mathrm{mb}$ and 1 for different values of $d$ and $i_{g}$ can be represented in logarithmetical scale by parallel lines with slope tangents $\longrightarrow 1$

tical line by means of a transparency. For this purpose an auxiliary line PQ (multiplication line) is drawn at random on the transparency (see FIG. 2). The transparency is placed on a graph of tilediameter lines with $i_{g}$ as parameter. The point at which the auxiliary line $P Q$ intersects the tile-diameter line $d_{1}$ with $i_{g}=0,10 \%$ is $C$. If with a constant $\mathrm{mb}$ and a diameter $\mathrm{d}$ one changes over from a slope of $0,10 \%$ with a length $l_{1}$ to a slope of $0,20 \%$, the tile-diameter line over a horizontal distance $a=0,55 \log \frac{0,20}{0,10}$ will have to be moved from $C$ to $B$. The point of intersection of the perpendicular through $B$ with the horizontal axis shows the new value for $1_{2 .}$. The figure $0,20 \%$, viz. the new slope, is added to the point of intersection $A$ of this perpendicular with the auxiliary $P Q$. The part $C B$ is in permanent relation-

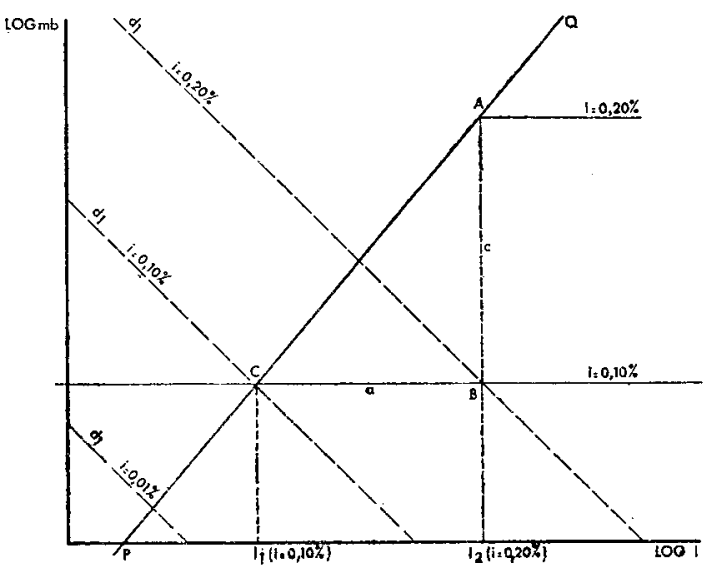

Fig. 2

As FIG. 1, but here the relationship between $\mathrm{mb}$ and 1 is read by means of the auxiliary line $P Q$ 
ship to $A B$, or $\frac{a}{c}=$ constant.

Vice versa, the length $c$ will be an index of the horizontal change a. A length $c$ and hence a length a correspond to each change in a slope of $0,10 \%$. The horizontal displacement over a distance $a$, in this example of $i_{g}=0,10 \%$ to $i_{g}=$ $0,20 \%$, is the same for all tile diameters.

In the displacement of a tile-diameter line $\mathrm{i}_{\mathrm{g}}=0,10 \%$ to $\mathrm{i}_{\mathrm{g}}=0,20 \%$ the triangle $A B C$ can therefore be placed at any desired position in the graph provided $C B$ is still parallel to the $\log 1$-axis and point $C$ lies on the point of intersection of the mb-line with the tile-diameter line corresponding to the slope $i_{g}=0,10 \%$. For this purpose a basic graph has been made in which the tile-diameter lines have been drawn which correspond to a slope of $0,10 \%$. Horizontal lines are drawn on the transparency in such a way that the distance from a given line $\left(i_{g}=0,10 \%\right)$ is equal to its accompanying new slope (see FIG. 3).

Fig. 3. Schematic representation of a part of the nomogram

- - - lines for the basic graph; lines for the transparency. In transition from, e.g., $0,10 \%$ to $0,20 \%$ the relationship $\frac{a}{c}=$ constant

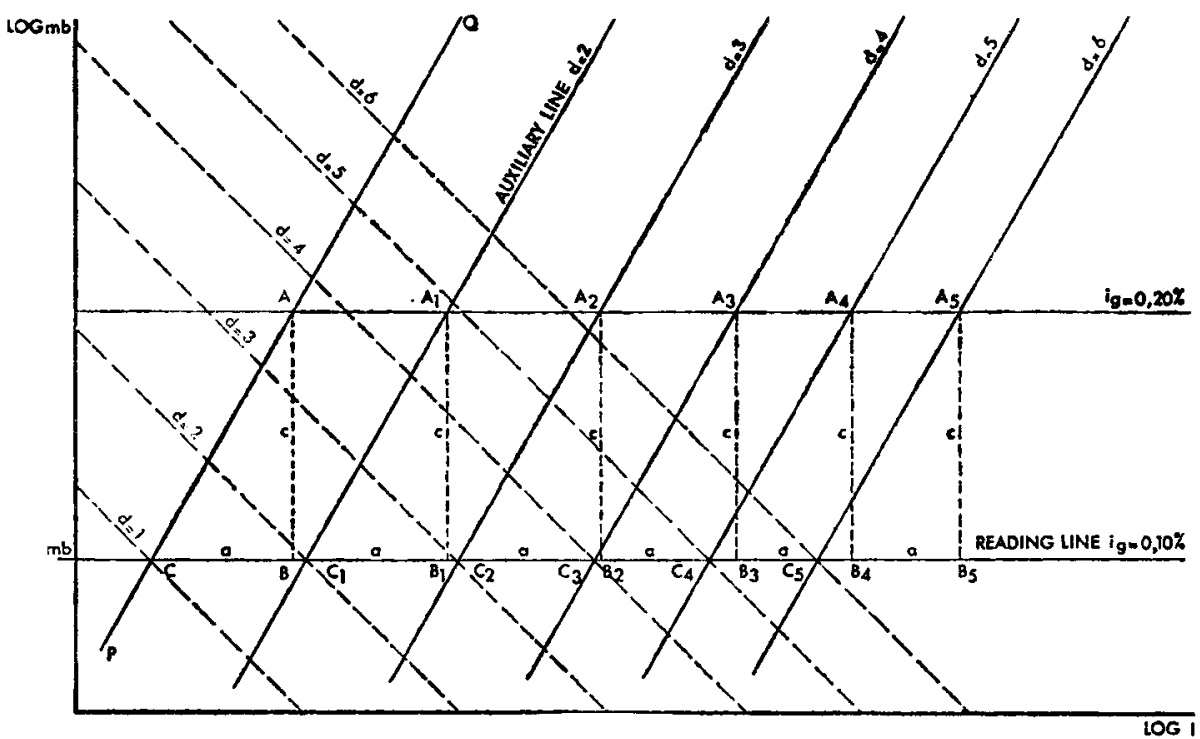

For the sake of simplicity, an auxiliary line is drawn on the transparency parallel to $\mathrm{PQ}$ for each tile-diameter line in the basic graph, thisauxiliary line intersects the tile-diameter line in question at a point in the common horizontal $i_{g}=0,10 \%$-line. This $i_{g}=0,10 \%$-line is called the reading line.

We thus obtain a transparent auxiliary graph in which the slope $i_{g}$ is shown on the vertical axis and auxiliary lines are drawn for the various tile-diameters. The $i_{g}=$ $0,10 \%$-line is clearly shown as a reading line because the basic graph has been cal- 
culated with this slope. As each auxiliary line belongs to a given tile-diameter line, the same notation is adhered to for both lines.

\section{Flow velocity}

The formula

$$
\mathrm{q}=\mathrm{mbl}=\mathrm{v} \cdot \mathrm{A}=\mathrm{v} \cdot 1 / 4 \pi \mathrm{d}^{2}
$$

enables the flow velocity to be calculated for every cross-section of a drain line filled with water.

Converted into the units used here, the equation becomes :

$$
\mathrm{mbl}=67,86 \mathrm{v} \cdot \mathrm{d}^{2}
$$

It follows from equations (2) and (3) that :

$$
v=\frac{2408}{67,86} d^{0,672} i_{g}^{0,55}
$$

or

$$
\log v=C+0,672 \log d+0,55 \log i_{g}
$$

in which $i_{g}$ is expressed in \% ;

$$
\begin{aligned}
& d \text { in } \mathrm{cm} ; \\
& v \text { in } \mathrm{cm} \cdot \mathrm{sec}^{-1} ; \\
& \mathrm{c}=\frac{2408}{67,86} \text {. }
\end{aligned}
$$

From this it follows that for a certain velocity the value of $d$ solely depends on the slope $i_{g}$ The horizontal and the vertical axis having been logarithmetically divided, straight lines can now be drawn on the transparency for constant flow velocities if the diameter is plotted along the horizontal axis. The scale on this axis has been so chosen that corresponding values are valid for the diameter in the points of intersection with the auxiliary lines.

$20,30,40,50$ en $60 \mathrm{~cm} \cdot \mathrm{sec}^{-1}$ have been selected as velocities.

\section{Method of using the nomogram}

The nomograms discussed and calculated above show the connection between tile diameter, geometric slope, drainage length and flow velocity for any desired case of drainage (see FIG. 7 and 8).

If the discharge intensity, drainage distance and the length are given, it is possible to calculate the tile diameter required, depending on the slope.

Example 1 (see Fig. 9)

$\mathrm{m}=10 \mathrm{~mm} \cdot(24 \mathrm{hrs})^{-1}, \mathrm{~b}=20 \mathrm{~m}$ and $1=200 \mathrm{~m}$.

Working method: Place the transparency with the reading line on the m.b-line of 200 (m.b). New read at $1=200 \mathrm{~m}$ what slope is required for the various tile diameters. Hence it follows that :

Tile diameter $4 \mathrm{~cm} ; \mathrm{i}_{\mathrm{g}}=0,20 \%$ decrease in piezometric head $(-\mathrm{h})=40 \mathrm{~cm}$

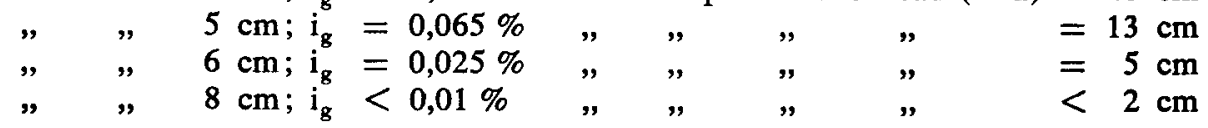


Hence in this case practically no slope is required in the line for an $8 \mathrm{~cm}$-tile, as a decrease of piezometric head of less than $2 \mathrm{~cm}$ is needed to obtain the drainage desired.

The height to which the flow velocity rises at the end of the line can also be ascertained by reading the flow velocities on the transparency for the various tile diameters at a length of $1=200 \mathrm{~m}$.

The diameter $4 \mathrm{~cm} ; \mathrm{v}=37 \mathrm{~cm} . \mathrm{sec}^{-1}$ with a slope of $0,20 \%$

$$
\begin{aligned}
& \text { " " } 5 \mathrm{~cm} ; \mathrm{v}=24 \mathrm{~cm} \cdot \mathrm{sec}^{-1}, ", \quad, 0,065 \% \\
& \text { " " } \quad 6 \mathrm{~cm} ; \mathrm{v}=17 \mathrm{~cm} \cdot \mathrm{sec}^{-1}, ", \quad ", \quad, \quad, 0,025 \%
\end{aligned}
$$

Note

If the drainage line is laid horizontally in such a way that the lowest point in the drain remains in situ, these flow rates of flow would still be reached. The necessary difference in piezometric head will build itself up over the drain.

The drawback of reading from the nomogram with a mb $=200$ is that small slopes have to be read outside the nomogram. It is better to take the reading on the $\mathrm{mb}=2000$-line, in which case there is no need to look vertically at $1=200 \mathrm{~m}$, but at $1=20 \mathrm{~m}$.

Consequently mbl would appear to be constant.

\section{Exam ple 2}

Assume a drain line to consist of $5 \mathrm{~cm}$-tiles with a slope of $0,20 \%$. At what discharge will the average piezometric slope be equal to the geometric slope if the drain ends in the free atmosphere when $b=20 \mathrm{~m}$ and $1=200 \mathrm{~m}$ ?

The transparency is placed on the basic graph in such a way that the points of intersection of the reading line and the auxiliary lines are on the points of intersection of the tile-diameter line with a possible mb-line.

The points of intersection on the transparency are then moved along the tile-diameter line in such a way that the intersection point of the $5 \mathrm{~cm}$ auxiliary line with $i_{\mathrm{g}}=$ 0,20 lies on the $1=200 \mathrm{~m}$-line. The reading line then shows the required $\mathrm{mb}$-value from which it is possible to calculate $\mathrm{m}$. In this case $\mathrm{mb}$ is 380 and $\mathrm{m}$ is $19 \mathrm{~mm}$ $(24 \mathrm{hrs})^{-1}$. If $\mathrm{m}$ is given, $\mathrm{b}$ can be calculated.

\section{Determining a collector drain with various tile diameters}

The relationship between the length and the piezometric head at any required diameter can be read from the nomogram. For water-removing drains no linear relationship exists between lengt 1 and piezometric head $h$, but according to equation (2)

so that

$$
1 \div \mathrm{i}_{\mathrm{g}} \mathbf{0 , 5 5}=\frac{\mathrm{h}^{0,55}}{1^{0,55}}
$$

$$
1 \div h^{0,355}
$$

In the case of a water-removing collector drain consisting of different diameters and lying on a given geometric slope it is therefore impossible to read off the tile diameters at once from this nomogram. In this nomogram, with regard to water-removing drains one should always start from the beginning of the drain line. When there is a linear relationship between 1 and $h$, as in the case of water-transporting conduits, the average piezometric slope $\bar{i}_{p}$ is the same for each longitudinal interval and should therefore be equal to the geometric slope $i_{g}$ at which the tiles have to be laid. 
FIG. 4 shows the piezometric head as a function of length 1 with a constant diameter for both types of transport. The FIG. shows that when the length for the $5 \mathrm{~cm}$ tile is read off from the nomogram for a given geometric slope in the direction of $l_{2}-l_{1}$, the average piezometric slope $\bar{i}_{p}$ of this part is greater than the geometric slope chosen.

When a line consists of different tile diameters (FIG. 5), the difference in piezometric head, as regards the nomogram, will have to be calculated for each length of a given diameter by determining the difference in piezometric head for each diameter

FIG. 4. Course of piezometric head lines of water withdrawal and water conveyance

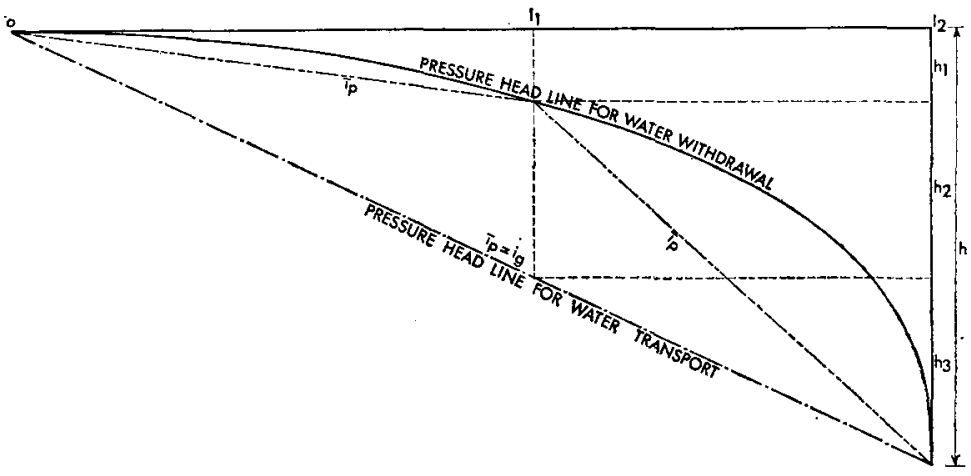

FIG. 5. Scheme for course of piezometric head in the case of compound drainage

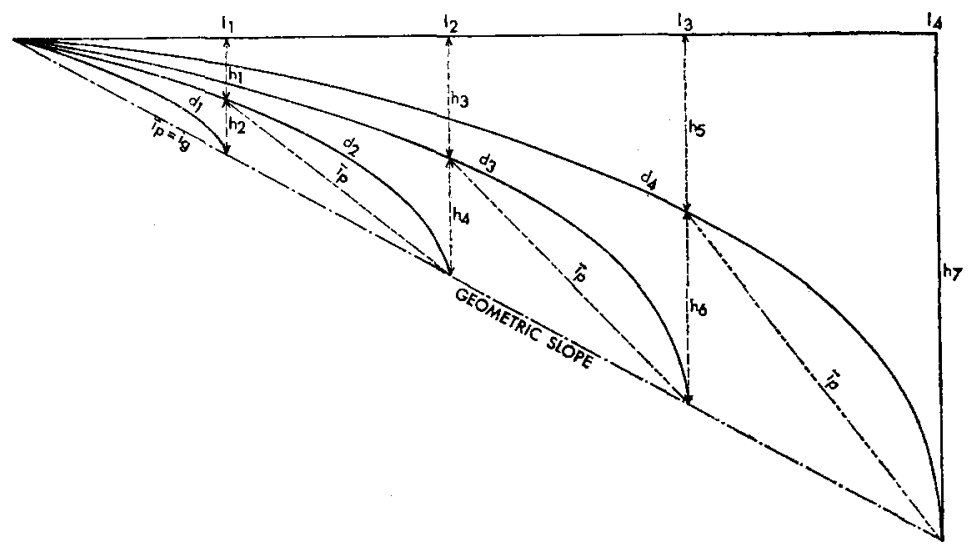

$d_{1}: 0$ to $1_{1}$ difference in piezometric head $=h_{1}+h_{2}$

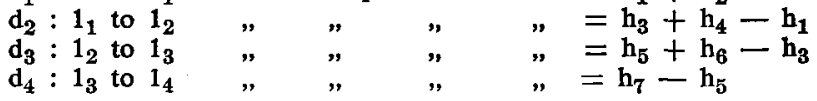

The difference in pressure head over the entire length of the series is now: $h_{2}+h_{4}+h_{6}+h_{7}$. From this it follows that the average piezometric slope, viz. $\frac{h_{2}+h_{4}+h_{6}+h_{7}}{1_{4}}$, is greater than the geometric slope, viz. $\frac{h_{7}}{1_{4}}$, at which the tile would be laid. 
for the total length, e.g. $\mathrm{l}_{1}$ and $\mathrm{l}_{2}$ in regard to a $2 \mathrm{~cm}$-tile and for the total length $l_{2}$ and $l_{3}$ for a $3 \mathrm{~cm}$-tile, etc.

Hence the composition of a collector drain found by reading the lengths for the different tile diameters in the nomogram horizontally along the line of slope would require a much greater difference in piezometric head than the determined geometric slope apparently calls for.

The following considerations show that a collector drain of different tile diameters can be composed by a method of approximation with regard to the nomogram. For this purpose we first calculate the length for the first tile diameter at the desired geometric slope after which the lengths of the remaining tile diameters are read off to the $8 \mathrm{~cm}$-line along the accompanying flow-velocity lines.

For tile diameters greater than $8 \mathrm{~cm}$ one can again read off the values along a line of slope belonging to the $8 \mathrm{~cm}$-tile. When, moreover, one diameter is repeatedly traversed (e.g. 4,6 and $8 \mathrm{~cm}$ or 5,7 and $9 \mathrm{~cm}$ ) the average piezometric slope over the whole length will be more favourable and even smaller than the geometric slope.

The equation (1), viz: $\mathrm{mbl}=2408 \mathrm{~d}^{2,672} \mathrm{i}^{0,55}$, can be written

$$
\mathrm{h}=-\left(\frac{\mathrm{mb}}{2408 \mathrm{~d}^{2,672}}\right)^{1,82} \cdot \mathrm{l}^{2,82}=\Gamma^{1,82} \cdot \mathrm{1}^{2,82}
$$

wherein $\Gamma=\frac{\mathrm{mb}}{2408 \mathrm{~d}^{2,672}}$.

The difference in piezometric head between the lengths $l_{1}$ and $l_{2}\left(l_{2}>l_{1}\right)$ of the same tile diameter is :

$h_{2}-h_{1}=-\Gamma^{1,82}\left(l_{2}^{2,82}-1_{1}^{2,82}\right)$ so that the partial slope $i_{p}$ becomes

$\mathrm{i}_{\mathrm{p}}=-\frac{\mathrm{h}_{2}-\mathrm{h}_{1}}{\mathrm{l}_{2}-\mathrm{l}_{1}}=\Gamma^{1,82}\left(\frac{\mathrm{l}_{2} 2^{2,82}-\mathrm{l}_{1}^{2,82}}{\mathrm{l}_{2}-\mathrm{l}_{1}}\right)$

or $i_{p}=\Gamma^{1,82}\left(\frac{1_{2}^{2,82}-1_{1}^{2,82}}{l_{2}-1_{1}}\right)$

$\left(\frac{l_{2}^{2,82}-l_{1}^{2,82}}{l_{2}-l_{1}}\right)$ must be constant for a determined $i_{p}$ and $\Gamma$.

Let us assume that $\frac{12^{2,82}-1_{1}^{2,82}}{l_{2}-1_{1}}=\lambda 1,82$

in which $\lambda 1,82=\frac{i_{p}}{\Gamma^{1,82}}$ or $\lambda=\frac{i_{p} 0,55 \cdot 2408 \mathrm{~d}^{2,672}}{\mathrm{mb}}$

Note

It follows from equation (7) that $l_{1}$ and $l_{2}$ can be interchanged without changing the equation. The line for which $l_{1}=l_{2}$ is thus a symmetrical line. If, therefore $l_{2}>1_{1}$ before the change is made it will have to be $\mathrm{l}_{1}>\mathrm{l}_{2}$ after the change.

FIG. 6 shows the relationship on the right-hand side between $l_{1}$ and $l_{2}$ for different values of $\lambda$. On the symmetrical line $\lambda=2,82^{0,55} 1$.

For $l_{1}=0$ (c.q. $l_{2}=0$ ) is $\lambda=1_{2}$ (c.q. $\lambda=l_{1}$ ), so that the values for $\lambda$ can also be read on the axes. The left-hand part shows the relationship between $\lambda$ en $\mathbf{d}$ according to equation (8) for $m b=1000$ and $i_{p}=0,10 \%$. Lines have also been drawn 


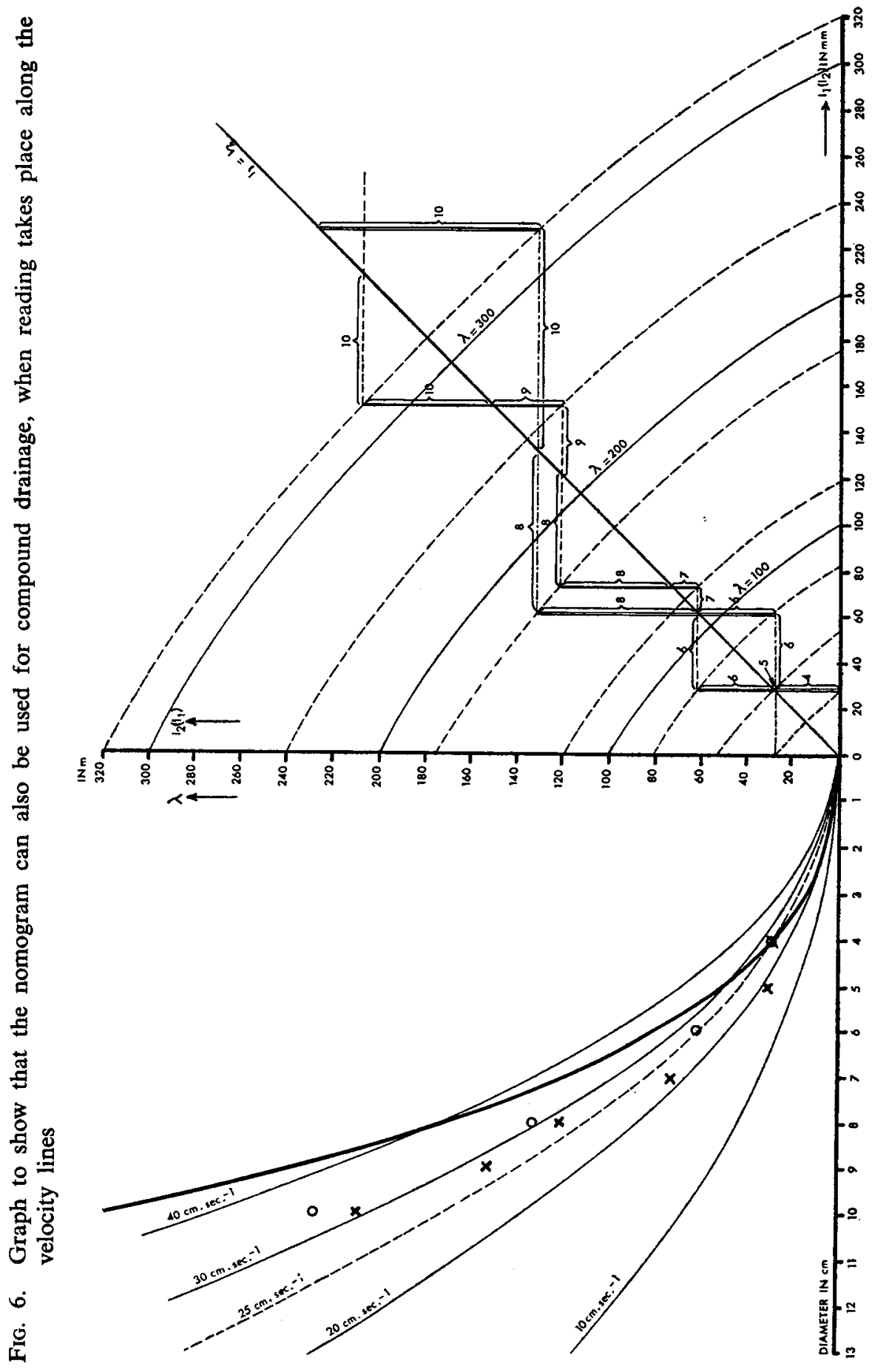


8

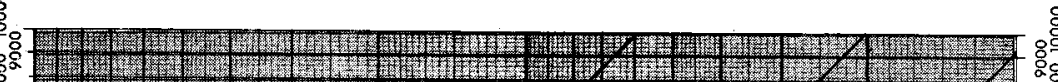

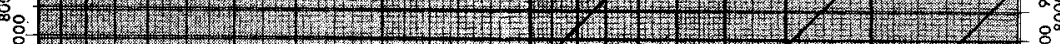

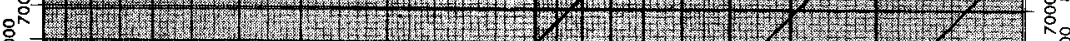

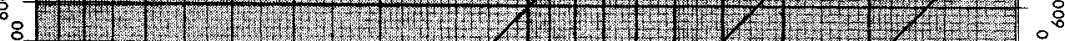

8 .

:

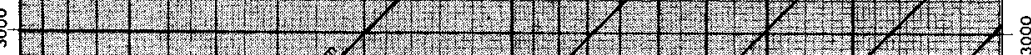

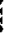

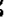

告

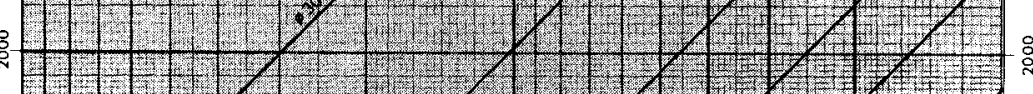

疍

.

$B$

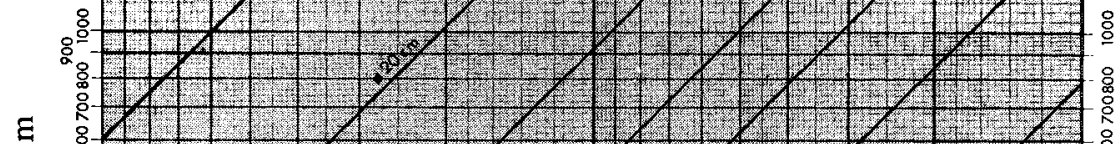

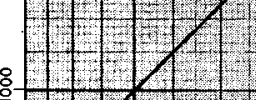

E

1.t.

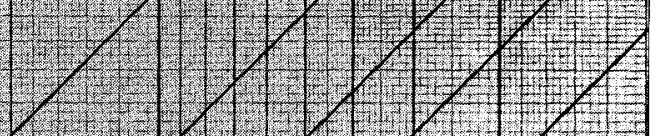

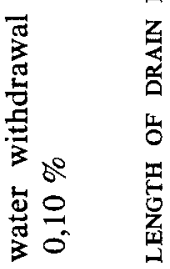

$\stackrel{0}{ } 11$

5

$\infty$

它

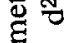

莺

용

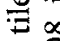

눈

등 11

离茝

$\therefore \quad \xi$

‥.

这 ह

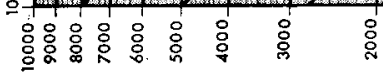

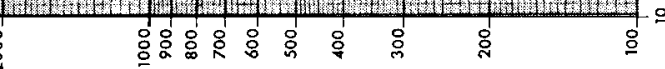


in this part for constant flow velocities, calculated according to equation (3), viz. $\mathrm{mbl}=67,86 \mathrm{v} \cdot \mathrm{d}^{2}$.

Commencing with a tile diameter of $4 \mathrm{~cm}$, a value for $\lambda$ is determined from the figure on the as in the case of the first part of the drain series $1_{1}=0$, it follows that $l_{2}=\lambda=27,7 \mathrm{~m}$. For the second part of the drainage series consisting of $5 \mathrm{~cm}$ tile we read $\lambda=50,4 \mathrm{~m}$.

The beginning of the new line is at $1_{1}=27,7 \mathrm{~m}\left(1_{2}\right.$ has become $\left.=1_{1}\right)$ and at $\lambda=$ 50,$4 ; 1_{2}=28,8 \mathrm{~m}$ is read from the end.

For a tile diameter of $6 \mathrm{~cm} \lambda=81 \mathrm{~m}$ and $l_{1}=28,8 \mathrm{~m}$ from which it follows that $l_{2}=60 \mathrm{~m}$ etc.

If the flow velocities corresponding to these lengths are shown in the left-hand part of FIG. 6, it appears that the flow-velocity points as regards the 5,6 and $7 \mathrm{~cm}$-tile lie below the flow-velocity line which passes through the point of the $4 \mathrm{~cm}$-tile. This means that if one reads along the flow-velocity line in the nomogram, for the $4 \mathrm{~cm}$ tile and $i_{g}=0,10 \%$, the lengths for the 5,6 and $7 \mathrm{~cm}$-tile are too great and therefore represent too great a piezometric slope.

If, in the same way, a compound line is determined in which one succeeding tile diameter is constantly traversed, it is found that the appropriate velocity points lie on or above the velocity line of the $4 \mathrm{~cm}$-tile. It is even found that in practically all cases beyond the $8 \mathrm{~cm}$-tile the velocity points are so far above the velocity line

FIG. 8. The transparent nomogram corresponding to the graph for drain tiles according to the formula $\mathrm{mbl}=2408 \mathrm{i}^{0,55} \cdot \mathrm{d}^{2,67}$ (see FIG. 7)

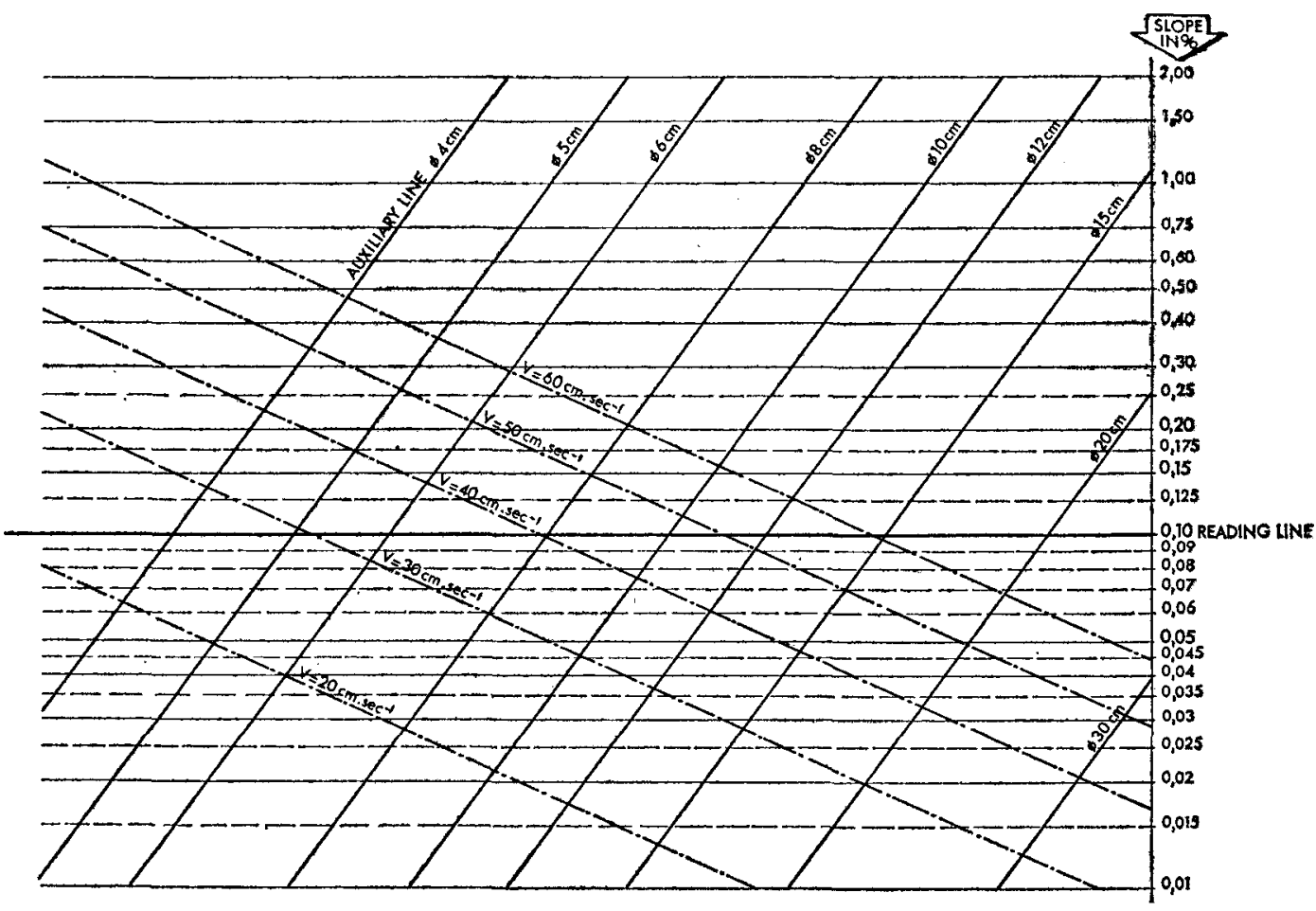

Neth. J. agric. Sci., Vol. 12 (1964) No. 2 (May) 
that if one reads the nomogram according to the flow-velocity line (i.e. the line corresponding to the geometric slope required for the first tile diameter) one can, without any objection, also read according to a line of constant slope for diameters $>8 \mathrm{~cm}$. In this case the partial piezometric slope is not greater than the geometric slope given.

\section{Final remarks}

With the aid of the nomogram ${ }^{1}$ described above it is now possible to determine quite simply all the factors which determine the design of a drainage system, e.g. diameters, geometric slope, the discharge intensity, drainage distance and flow velocity in their mutual relationship.

By these means it is possible, on a basis of the definite data, to choose for each drainage problem the solution, which provieds the best answer to the requirements made on it. Although the nomogram was not primarily constructed for the calculation of compound drains, it is nevertheless possible to determine the individual lengths for the various tile diameters by means of approximation.

\section{REFER E N C E}

VISSER, W. C.

1954 Tile drainage in the Netherlands. A summary of the addresses delivered on the tile drainage day, 16th May, 1952. Neth. J. agric. Sci. 2. $2: 69-87$.

1 The nomogram with transparency size $40 \times 30 \mathrm{~cm}$ can be supplied by the Research Dept. of the Koninklijke Nederlandsche Heidemaatschappij, Arnhem, Netherlands, for the sum of Dfl. 25,-- 


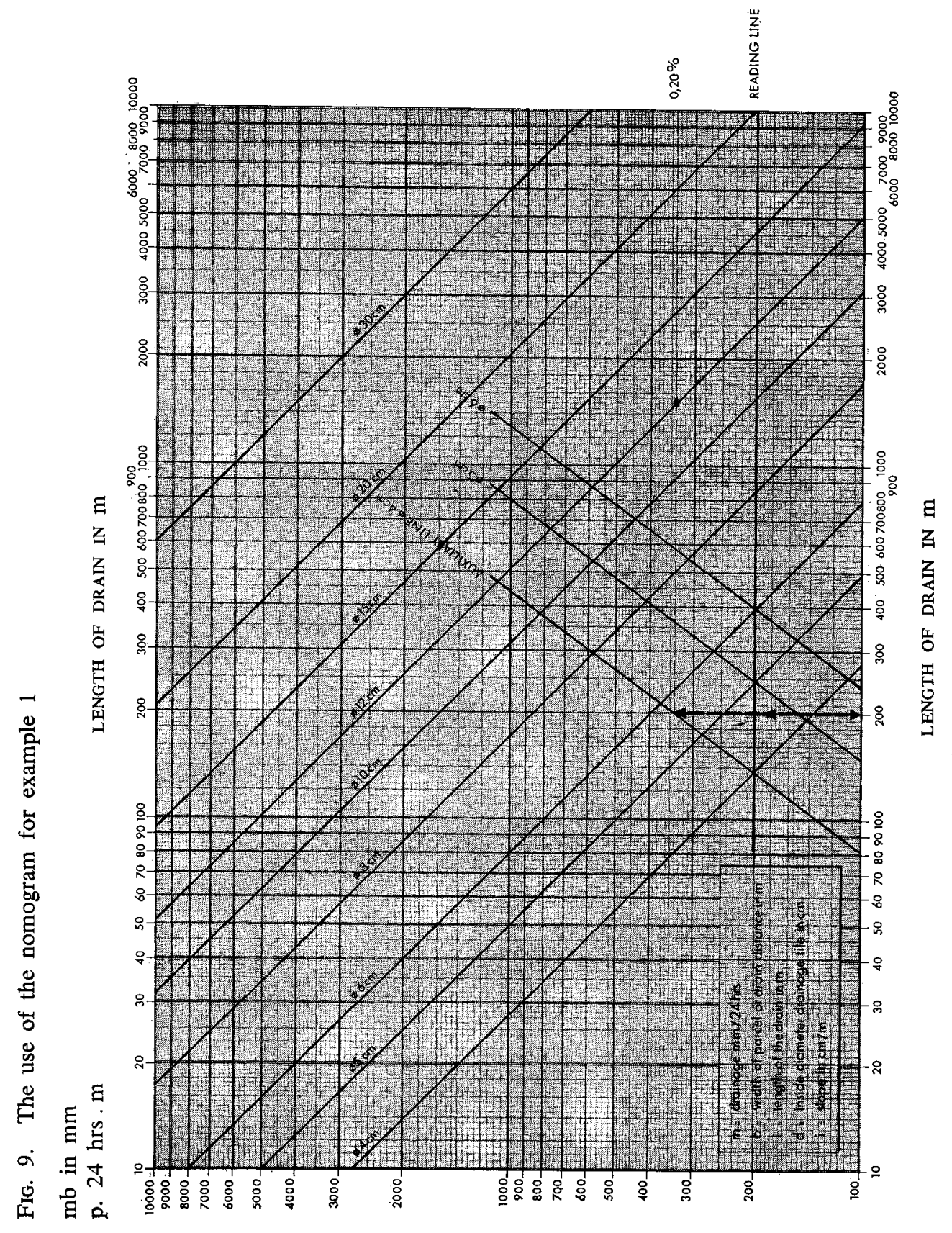

